\title{
Fuzzy Based Mobility Management in 4G Wireless Networks
}

\author{
M Phemina Selvi ${ }^{1}$, S.Sendhilnathan ${ }^{1}$. \\ ${ }^{1}$ Assistant Professor/ Dept. of ECE, University College of Engineering Villupuram.
}

ABSTRACT

Handover is the key procedure in wireless networks to provide required qual ity of service to the users during mobility Handover. As the current scenario of wireless communication comprises of heterogeneity in architecture, application and radio access technologies concentrating on vertical handover becomes important to provide continuous communication and ubiquitous coverage. This paper provides comprehensive survey of the vertical handover, decision technique and parameter used for making proper handover in horizontal and vertical handover. The recent handover schemes are discussed and classified which varies based on concepts and the adopted schemes. A Fuzzy based decision making algorithm is proposed to made the handover decision more appropriate and to avoid loss of communication and provide better performance.

Key words: 4G, Mobility Management, Heterogeneous Networks, Vertical Handover, Fuzzy.

\footnotetext{
*Author for correspondence: sendhilnathan29@yahoo.co.in
} 


\section{INTRODUCTION}

In recent years, there is a paradigm shift in the development of various wireless technologies. Wide variety of wireless networks has emerged, which lead to heterogeneous network environment. Wireless user population is growing sharply, due to the increase in multimedia services like streaming media, audio and video in live and other social networking services, expecting seamless communication anytime, anywhere with the upgraded multimode user terminals. No single access technology or mobile network can ever meet these requirements of the current wireless scenario of providing seamless communication without loss and delay with the available bandwidth. The $4 \mathrm{G}$ or the next generation wireless network comprises of mixed networks such as $2 \mathrm{G}, 3 \mathrm{G}$, wireless LAN (WLAN), IEEE 802.16e (WiMaX) etc. each differing in bandwidth capacity, coverage area, network architecture, routing protocol, transport protocols, data rates, mobility management and operational costs. The paradigm of $4 \mathrm{G}$ networks are designed to provide superior performance in terms of quality of service, data transmission speed, reduced latency, high security , support integrated services and also to provide higher bandwidth, higher data rate and low authentication overhead with seamless connectivity. ${ }^{1}$ The $4 \mathrm{G}$ networks are envisioned towards convergence / integration of various access radio access networks. 4G is referred as MAGIC- Mobile Multimedia, Anytime anywhere, Global mobility support, Integrated wireless solution and Customized personal service. Various challenges are to be addressed during the integration of heterogeneous networks classified as wireless local area networks (WLAN), wireless personal area networks(WPAN), wireless metropolitan Area Networks (WMAN) and wireless wide area networks(WWAN). All the networks are operated under all IP networks. One of the major challenges to be addressed in heterogeneous networks is mobility management. Any mobile user (MU) or mobile host (MH) or mobile terminal (MT) seeks seamless access of internet while on the move without any disruption. The evolution of different wireless technologies, its growth and deployment is provided in Table 1.

Different networks co-exist to satisfy the requirement of their users, but they behave complementary to each other in terms of their capabilities and support for different applications. Integrating these networks

enables the mobile users to be always connected to the best access network among the various networks available depending on their requirements. Integration of heterogeneous networks leads to heterogeneities in access technologies, bandwidth, transmission rate, transmission power requirement, coverage area and network protocols. In order to provide the requirements of mobile users under this heterogeneous environment, a common infrastructure required to interconnect multiple access networks is needed which supports data accession anywhere at any time. For efficient delivery of servic es to the mobile users, the future generation wireless networks require new methods of mobility management where the location of every user is proactively determined before the service is delivered. Moreover, for designing an adaptive communication protocol, various existing mobility management schemes are to be seamlessly integrated. Efficient handover mechanisms are necessary for ensuring seamless connectivity and uninterrupted service delivery 2 . 
Table1: Growth of Wireless Communication

\begin{tabular}{|c|c|c|c|c|c|}
\hline & $1 \mathrm{G}$ & $2 \mathrm{G}$ & $2.5 \mathrm{G}$ & $3 \mathrm{G}$ & $4 \mathrm{G}$ \\
\hline $\begin{array}{l}\text { Year } \\
\text { Introduced }\end{array}$ & 1985 & 1992 & 1995 & 2002 & 2010 \\
\hline Frequency & $\begin{array}{l}400 \mathrm{MHz}- \\
800 \mathrm{MHz}\end{array}$ & $\begin{array}{l}400 \mathrm{MHz}- \\
900 \mathrm{MHz}\end{array}$ & $1800 \mathrm{MHz}-1900 \mathrm{MHz}$ & $\begin{array}{l}400 \mathrm{MHz}- \\
900 \mathrm{MHz}\end{array}$ & $3 \mathrm{GHz}-5 \mathrm{GHz}$ \\
\hline $\begin{array}{l}\text { Driven } \\
\text { Technique }\end{array}$ & Analog & Digital & Packet switching & $\begin{array}{l}\text { Intelligent signal } \\
\text { processing }\end{array}$ & $\begin{array}{l}\text { Auto configuration } \\
\text { Intelligent software }\end{array}$ \\
\hline Standards & $\begin{array}{l}\text { AMPS } \\
\text { NMT, } \\
\text { TACS } \\
\end{array}$ & GSM & $\begin{array}{l}\text { GPRRS,EDGE,HSCSD,I- } \\
\text { Mode }\end{array}$ & $\begin{array}{l}\text { IMT2000(UMTS, } \\
\text { WCDMA } \\
\text { CDM A2000 }\end{array}$ & OFDM,UWB \\
\hline $\begin{array}{l}\text { Multiple } \\
\text { Access } \\
\text { Techniques }\end{array}$ & FDMA & TDM A,CDMA & TDMA,CDMA & CDMA & FDM A,TDMA.CDMA \\
\hline $\begin{array}{l}\text { Cellular } \\
\text { Coverage }\end{array}$ & $\begin{array}{l}\text { Large } \\
\text { area }\end{array}$ & Medium area & Medium area & Small area & Very Small area \\
\hline $\begin{array}{l}\text { Bandwidth } \\
\text { Kbps }\end{array}$ & $\begin{array}{l}2.4 \mathrm{Kbps}- \\
30 \mathrm{Kbps}\end{array}$ & 9.6-14.4 Kbps & 171-384 Kbps & 2- $5 \mathrm{Mbps}$ & 10- $20 \mathrm{Mbps}$ \\
\hline $\begin{array}{l}\text { Core } \\
\text { Networks }\end{array}$ & $\begin{array}{l}\text { Telecom } \\
\text { Networks }\end{array}$ & $\begin{array}{l}\text { Telecom } \\
\text { Networks }\end{array}$ & Telecom Networks & $\begin{array}{l}\text { Telecom } \\
\text { Networks and } \\
\text { some IP networks }\end{array}$ & All IP Networks \\
\hline $\begin{array}{l}\text { Services } \\
\text { Supported }\end{array}$ & Voice & Voice, SMS & Data Service & $\begin{array}{ll}\text { Voice, Data, } & \text { Few } \\
\text { multimedia } & \text { man } \\
\text { to machine } & \end{array}$ & $\begin{array}{l}\text { Multimedia machine to } \\
\text { machine. }\end{array}$ \\
\hline
\end{tabular}

Mobility management is classified into location management and Handover management. Location management is concerned with updating the user current position for delivery of data without service failure. Handover/handover Management is done while the user is in roaming i.e. when the mobile terminal moves from one access point/ base station in one access domain to another access point/ base station in another access domain. Handover management refers to the process of transferring a mobile user between cells of the same or a different network without disrupting connections 3 . When heterogeneity is of prime consideration then the important management to be addressed is Handover/handover management. Various proposals have been done for the handover management.

Different handover management techniques are proposed by different research groups in heterogeneous networking scenarios. Mobile internet protocol is the basic mobility protocol which supports mobility. So the core network must be an IP based network and the technologies used must be an IP based technologies to achieve seamless communication. Several commercial agencies and research sectors are working towards the area of wireless networks that support universal roaming across multiple wireless and mobile networks - for example, from a cellular network to a satellite-based network to a highbandwidth wireless LAN. We need a system where roaming is seamless and users are always connected to the best network.

This paper is organized as follows. Section 2 provides a brief description on the challenges and requirements of $4 \mathrm{G}$ networks and concepts of mobility management. Section 3 discusses handover strategies while Section 4 gives an overview of handover issues and various proposed algorithms and their effects 
and the proposed fuzzy decision system with its discussion and concludes the paper with the remarks.

\section{SECTION II}

\section{CHALLENGES OF 4G WIRELESS NETWORKS}

The key concept of $4 \mathrm{G}$ is integration of different networks. The main challenges comprises

terminal network, application and user/Mobile terminal/ host ${ }^{4}$.Conceptually, a $4 \mathrm{G}$ wireless network architecture can be viewed as many overlapping wireless Internet access domains. In this heterogeneous environment, a mobile host (MH) is equipped with multiple (often called multi-mode) wireless interfaces to connect to any or all wireless access networks anytime anywhere. Therefore, providing seamless mobility support without disruption is one of the most challenging problems for the system integration in $4 \mathrm{G}$ wireless networks.

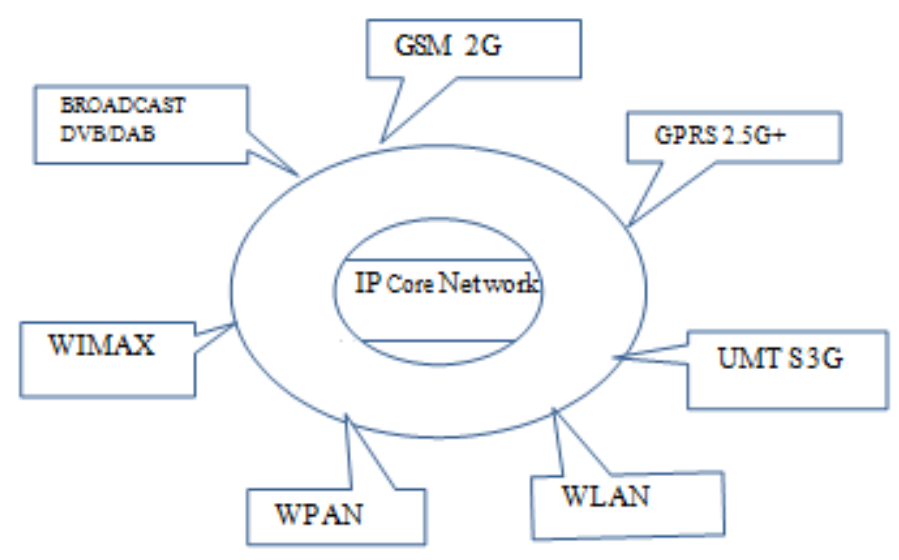

Figure 1: Conceptual Diagram of $4 \mathrm{G}$ Network

The convergence of wireless access has paved a path to establish 4G network with IP has the core network. Convergence in terminals, networks and application may also be referred as seamless integration which is the goal of 4G. Any network technology change requires minimal changes in the existing technology which must be backward compatible. The development of $4 \mathrm{G}$ is to facilitate superior quality and data transmission speed compared to $3 \mathrm{G}$, so that it can support multimedia service including video more effectively with a data transfer rate of $100 \mathrm{Mbps}$ minimum, for Mobile Host travelling at very high speed \&data transfer rate of one Giga bit/sec for a fixed /stationary Mobile Host. The conceptual diagram of any $4 \mathrm{G}$ networks is illustrated in Figure 1.

\section{REQUIREMENTS OF 4G}

1. $4 \mathrm{G}$ network must provide high quality \& better data transmission speed.

2. It must possess an environment which supports rapid transmission speed an requires appropriate

Security measures which can support high data transmission with data quality and integrity preserved.

3. The user must be capable to access internet anytime \&anywhere with Always Best Connected (ABC).

4. Requires an approach that enhances quality for multimedia services.

5. Also it requires a mechanism that integrate IP based Non IP based services 
6. It requires a novel mechanism for the mobile host that can operate in different network. i.e. Design of

multimode terminal for supporting $4 \mathrm{G}$ services and has to address the problem of system discovery

and selection.

\section{REQUIREMENTS OF MOBILITY MANAGEMENT}

It must support all forms of mobility and support mobility for all application. It must support mobility across heterogeneous networks and must support session continuity with a support for Support global roaming. The Basic functional capabilities of Mobility management are

Location Management: The location management enables to determine mobiles current point of attachment, Packet delivery to mobile using the location information to deliver the packets, Handover and Roaming while the mobile terminal changes the network point of attachment on the move and Network Access Control which does the work of (AAA) Authentication, Authorization and for security.

Handover Management: Handover is a term used to represent a situation in which an ongoing voice call or data transmission is handed of over from one interface i.e. AP/BS in WLAN /Cellular network to another interface BS/AP without disrupting the ongoing communication. The term handover is also referred as handover in heterogeneous wireless networks. Handover management does the operation of maintaining the ongoing communication seamless without any discontinuity in service while the mobile user is on the move, changing its point of attachment. Different scenarios of handover are, i. When the mobile node moves from one point of attachment to another in the same radio access technology, which means homogeneous network, ii. When the mobile node moves from one point of attachment to another point of attachment of different radio access technology which means heterogeneous network, iii. When the current point of attachment of the mobile node is not capable of satisfying the user quality of service. Since the wireless networks are random in nature, continuous monitoring and controlling of handover process is necessary in handover management to provide an uninterrupted service. The handover can be performed either by the mobile or the network. Based on this handover strategies are classified as Mobile controlled handover (MCHO), mobile assisted handover (MAHO), network controlled handover NCHO) and network assisted handover (NAHO). These classifications are based on whether the mobile node initiates the handover or network initiates the handover ${ }^{8}$. The handover processes are also classified as proactive and reactive.

\section{TYPES OF HANDOVER}

Handover is classified as hard handover \& soft handover based on whether the connection is break before make or make before break respectively .But choosing of hard handover or soft handover must be done considering the resource efficiency and ping pong effect. Further handover is classified as horizontal handover \& vertical handover. Horizontal handover [Intra-domain handover] is a process in which the handover occurs inside the same radio access network i.e. same access technology. Vertical handover (Inter-domain handover] is a process in which handover/handover occurs from one radio access network to different access network i.e. from 3G UMTS to GSM or from GSM to WLAN. It is also referred as Media Independent Handover (MIH) and has been standardized as IEEE 802.21. 
Vertical handover is done in three phases. The first phase is the Network discovery/System discovery, which is done by gathering all the network related information of nearby networks to identify the need for handover. Second phase is Handover Decision, which is done with help of measured network parameter / metrics taking some specific criterion into account to choose an appropriate network.

To perform intelligent and better decision in choosing a wireless network for proper delivery of required service that is most suitable for it, additional metrics like service type, network conditions such as available bandwidth and network access delay, system performance mobile node capabilities, user preferences, monetary cost with RSSI are parameters both for horizontal and vertical handover as in traditional methods. The third phase is Handover Execution, which performs the handover at the right time. The mobility management problems have been addressed at different layers using newly developed protocols. Session Initiation protocol(SIP) supports mobility management in application layer, the Stream control transmission protocol(SCTP) in Transport layer, Host identity protocol in the intermediate layer between network and transport layer, mobile IP , Fast mobile IP (FMIP), HMIP,FHMIP supports mobility management in network layer.

\section{SECTION III}

\section{HANDOVER STRATEGIES}

Various handover decision strategies have been proposed to determine whether handover is required or not, to select a suitable network based on user requirement if handover is necessary, and how to execute the handover and when to perform handover. To decide whether a handover is required or not, various deciding parameters are used in addition to the conventional parameter RSS .To decide vertical handover the following process are required.

1. The sequence of decision process.

2. Defining criteria's used for handover decisions.

3. Gathering the criterion information.

4. Determining the handover policies to apply.

5. Decision making either by network or Mobile Node.

6. Optimization of handover performance if necessary.

According to handover initiation, vertical handover is classified as imperative or forced handover and Alternate or user handover. Imperative or forced handover is initiated by obtaining the physical event information about the availability of the network interface. Alternate or user handover is done by using user performance or policies using policy parameters or metrics like money, cost, parameter regarding QOS criteria, set of rules has to be written, for how and when handover is to be triggered, by selecting the best access network to switch seamlessly.

\section{DECISION METRICS FOR HANDOVER}

The metrics for deciding handover are those which give information of whether a handover is required or not are classified into four categories, network related parameters, terminal or node related parameters, user related and service related parameters. They are also classified as static and dynamic in context of frequency and changes in metrics. Network related metrics are coverage, bandwidth, latency, link quality (RSS), Carrier to Interference Ratio(CIR), Signal to Interference Ratio(SIR), Bit Error rate (BER),monetary cost, 
security level. Terminal related metrics are velocity, power consumption, location information are the user related metrics are the user preferences and user profile. Service related metrics are QOS and service capabilities.

\section{DECISION POLICY OF HANDOVER}

The decision policy decides to choose which access network and when to perform the handover. In traditional handover, RSS plays the major role in decision policy. Depending upon the old measured RSS, comparing the handover is done. The other decision is based on dwell time to perform handover. Many strategies have been proposed in the literature for vertical handover decision. They are broadly categorized into five

1. Function based strategies

2. User centric strategies

3. Multi criterion based strategies

4. Fuzzy logic and neural network based strategies

5. Context aware strategies

\section{PERFORMANCE METRICS}

Performance metrics are used to quantitatively evaluate the vertical handover decision algorithm that is proposed under various network scenarios, example the network considered may be two/more radio access network like WLAN, WWAN, WMAN ( 802.11b to GSM/GPRS, WIFI+WIMAX+GPRS combination etc.,).The parameters necessary for the performance evaluation are 1.Handover delay

2. Number of handover 3.Probability of handover failure and 4.Throughput. By measuring the maximum delay in handover, number of handover required, number of failed handover and the overall throughput of the session, the performance of the any proposed algorithm can be analyzed for vertical handover. The procedure of handover and its major steps is provided in the Table 2.

Table2: Handover Operation

\begin{tabular}{|c|c|c|c|c|}
\hline \multicolumn{4}{|c|}{ HANDOVER } & \multirow{3}{*}{ A } \\
\hline \multicolumn{2}{|c|}{ INFORMATION GATHERING } & \multicolumn{2}{|c|}{ HANDOVER DECISION MAKING } & \\
\hline Network Related & Mobile Terminal Related & Criteria & Strategy & \\
\hline $\begin{array}{ll}\text { - } & \text { Link quality } \\
\text { - } & \text { Coverage } \\
\text { - } & \text { QOS } \\
\text { - } & \text { Cost }\end{array}$ & $\begin{array}{ll}\text { - } & \text { Velocity } \\
\text { - } & \text { Stations } \\
\text { - } & \text { User } \\
& \text { preferences } \\
\text { - } & \text { Velocity }\end{array}$ & $\begin{array}{ll}\text { - } & \text { RSS } \\
\text { - } & \text { Velocity } \\
\text { - } & \text { QOS parameters } \\
\text { - } & \text { User preferences } \\
\text { - } & \text { Battery usage } \\
\text { - } & \text { Operator } \\
& \text { performance } \\
\text { - } & \text { Security } \\
\text { - } & \text { Available RATs } \\
\text { - } & \text { Bandwidth }\end{array}$ & $\begin{array}{ll}\text { - } & \text { Traditional } \\
\text { - } & \text { Function based } \\
\text { - } & \text { User centric } \\
& \text { Based } \\
\text { - } & \text { Fuzzy logic based } \\
\text { - } & \text { ANN based } \\
\text { - } & \text { Multiple attribute } \\
& \text { based } \\
\text { - } & \begin{array}{l}\text { Context aware } \\
\text { based }\end{array}\end{array}$ & $\begin{array}{l}\mathrm{E} \\
\mathrm{X} \\
\mathrm{E} \\
\mathrm{C} \\
\mathrm{U} \\
\mathrm{T} \\
\mathrm{I}\end{array}$ \\
\hline
\end{tabular}

\section{VERTICAL HANDOVER DECISION}

The vertical handover decision is performed by using the algorithms. The algorithms are classified into four types based on the criteria used for deciding the handover. The major criteria's considered are RSS, bandwidth, cost function and combination of the 
three. In traditional handovers the received signal strength (RSS) is considered as the parameter for handover decision. But it is not sufficient for heterogeneous scenario because the RSS of different networks c a n n o t be compared directly. This type of conventional handover does not provide the network condition information which is the necessary to decide whether a handover is required or not, and to which network the handover must be performed.

A good vertical handover decision algorithm must be developed considering all the metrics such as service type, monetary cost, network conditions, system performance, mobile terminal conditions and user preferences with that of RSS measurement. Multi-criteria in the policy-based approaches are not only for deciding when the handover occurs but also determine which network should be selected as a target network and whether handover is worth ${ }^{5}$.In Bandwidth based algorithm, the bandwidth available for a mobile terminal is of prime concern. The cost function based algorithm uses metric related to cost in times of security, bandwidth, power consumption and monetary cost. A cost function is determined by assigning different weights to the metrics based on network condition and user preferences. Based on RSS, many algorithms developed for vertical handover decisions.

\section{BIRDVIEW OF VERTICAL HANDOVER}

Mohanty and Akyildiz's ${ }^{5}$ considered $3 \mathrm{G}$ and WLAN network for simulation. In their work the current RSS is compared with a dynamic RSS threshold to determine the handover time from WLAN to $3 \mathrm{G}$. Their algorithm provided a reduction in the false handover initiation and handover failure probabilities. However the work didn't support for the reverse scenario i.e. $3 \mathrm{G}$ to WLAN. A bandwidth-based vertical handover decision algorithm between WLAN and wideband CDMA (code division multiple access) networks have been proposed in $^{6}$ that uses the signal to interference and noise ratio (SINR) as its main criterion. This algorithm provides users with a higher overall throughput as well as a balanced load between the WLAN and the WCDMA network. However, this technique may introduce too much handover given the dynamic variations of the SINR and may result in a 'ping pong' effect between access points.

Ali cephal and celal cekan ${ }^{7}$ developed the Fuzzy logic based vertical handover decision making algorithm and an Adaptive Network Fuzzy Interface System(ANFIS) based vertical handover decision making algorithm combining RSSI, data rate and monetary cost as input and concluded that ANFIS algorithm out performs both for user and the network in reducing the handover and reducing decision time. Abolfazl et al ${ }^{8}$ proposed a fuzzy based vertical handover necessary estimation and the target network selection algorithm using predicted RSS and degree of provided QoS based on requested traffic class and speed of vehicle with the direction of mobile station.

Qing $\mathrm{He}$ et $\mathrm{al}^{9}$ proposed a vertical handover decision algorithm considering the RSS, available bandwidth user preference and monetary cost as the criteria for decision vertical handover. They used fuzzy based vertical handover decision algorithm for handover initiated by mobile networks and cost function based vertical handover initiated by point of attachment. When MN initiates handover it selects suitable network using fuzzy logic based vertical handover decision algorithm. When hand off is initiated by point of attachment, the cost function based vertical handover decision algorithm selects the MN with low cost function, and the selected MNs are given to the nearby point of attachment BS's or APs to balance the load. Qingyang song ${ }^{10}$ developed a new network selection scheme for an integrated network scenario combining of cellular and the WLAN systems that could provide the best QoS at anytime. They used analytical hierarchy process(AHP) to fix the weights of the criteria evaluated with reference to user preferences and service appreciation and GRA to evaluate the user preferences with necessary service classes. 
Mobility Management in 4G Wireless Networks

Wei Song \& Yu Cheng et $\mathrm{al}^{11}$ presents a policy framework for managing the resources ia a cellular WLAN integrated network is based on loose coupling. The scheme balances the multiservice traffic load over the two integrated network by using admission control and takes advantage of dynamic vertical handover to adapt to network state and variations in traffic load. Their work implies that high utilization of resources is achieved by dynamically balancing the offered traffic load over the two networks.

Sunisa Kunarak and Raungrong Suleesathira ${ }^{15}$ proposed intelligent algorithm for vertical handover decision which supports both for real time and non real time services and provides network selection for WLAN/WiMAX/UMTS considering predictive received signal strength of the target networks, received signal strength (RSS) of current serving network and residence time in the target network if their conditions are consistently true during dwell time to reduce connection dropping and unnecessary handovers. They have used back propagation neural network for predicting the RSS and fuzzy logic for network selection. Tansir Ahmed et al ${ }^{16}$ proposed an intelligent context aware vertical handover decision algorithm for multimode mobile terminals which takes the session transfer into account considering the intelligence both on terminal and network side which they term as context information. The algorithm is based on the Analytical Hierarchy Process (AHP) for which the inputs are defined using primary objectives for decision algorithm. The objectives defined in turn depend on the user preferences. The capabilities of the available networks are measures and checked for the suitability of the user preferences and the best suitable network is selected. This method suits only for average users, minimizing processing time, hand over delay and CPU and memory usage.

Vijay and Jeffery ${ }^{17}$ devised a mobility management procedure for UMTS and WLAN for different integration scenarios to achieve seamless mobility. The architecture considered for mobility management are the tight coupling, loose coupling and peer networks. They concluded that the peer networking is least complex ,tight coupling is little bit more complex then loose coupling which is most challenging.

Yang et. al. ${ }^{18}$ proposed an algorithm considering cellular networks and WLAN in which a dynamic threshold is calculated and compared with the predicted travelling time inside the WLAN to decide the handover in which handover failure rate was reduced ,but resulted in unnecessary handover and connection breakdown probabilities with increase in handover delay. Zahran et.al. ${ }^{20}$ proposed an algorithm for $3 \mathrm{G}$ and WLAN networks, which is based on RSS in combination with an Estimated lifetime of the available bandwidth to decide the time of handover, which enhances the bandwidth utilization.. But there is an increase in packet delay that increases with the lifetime of the connectivity due to the changes in the channel condition as the mobile Terminal approaches the edge of the WLAN cell. Adaptability to application requirements and user mobility is the merit of this work.

\section{PROPOSED WORK}

\section{SECTION IV}

The key contribution of the paper is proposing a fuzzy based vertical handover control decision for a heterogeneous network. The proposed vertical handover algorithm uses a Mamdani fuzzy logic inference system. It is composed of a fuzzifier, a fuzzy inference engine and a de-fuzzifier. A fuzzification changes input parameters from the crisp numbers into the fuzzy sets by appropriate membership functions. Then, fuzzy inference aggregates the analyzed fuzzy sets based on the pre-defined fuzzy rules. Finally, a crisp output for the aggregated value is given through the de-fuzzification process. The input metrics considered are Received Signal Strength (RSS), Bandwidth (BW), Monetary Cost(MC), User Preference(UP) and Velocity of the mobile user(V) for the networks considered. Each of the input parameters is transformed to one of three fuzzy sets (Low, Medium, and High) by the membership functions. The fuzzy inference system uses the max-min Mamdani implication method. Membership function 
is a curve that defines how each point in the input space is mapped to a degree of membership between 0 and 1 . The input space is also referred as the universe of discourse. The proposed work is shown in figure 3 .

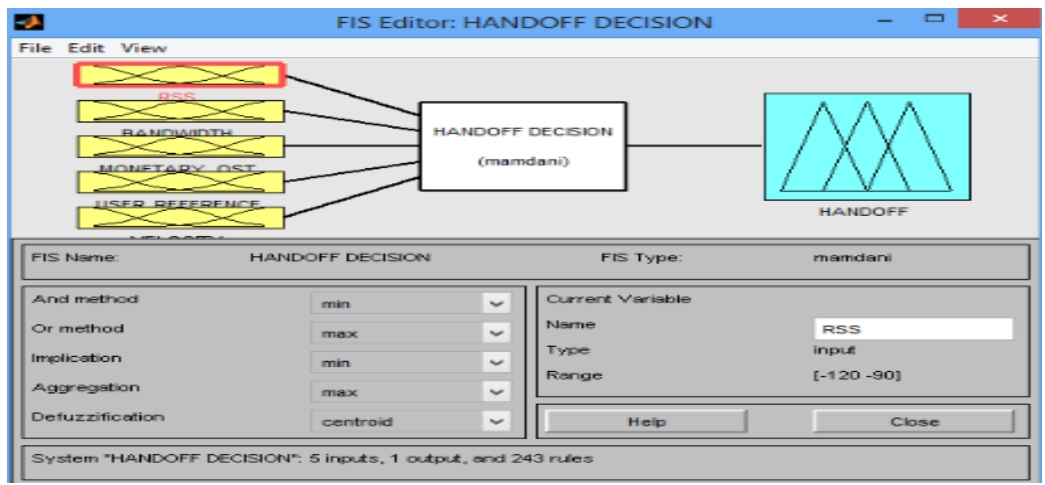

Figure 2. Screenshot of FIS for handover

The works previously done consider two or three input for making decision The proposed work considering five inputs may make the system more complex in deriving the menbership function but produces more better decision for vertical handover. Considering three input and wirting 27 rules will make the system easier and simple.As number of input increases the number of rules increases to 243 in which the decision can be made more precise in deternining the handover execution. Adjusting in the rule viewer the variation for the handover decision requirement can be analyzed and it is seen that as RSS decreases and bandwidth decreases the necessity of the handover required also increases this ruler view helps to view and analyze output parameter by changing the input parameters . The screenshots with five inputs fuzzy interference systems is shown in Figure2.

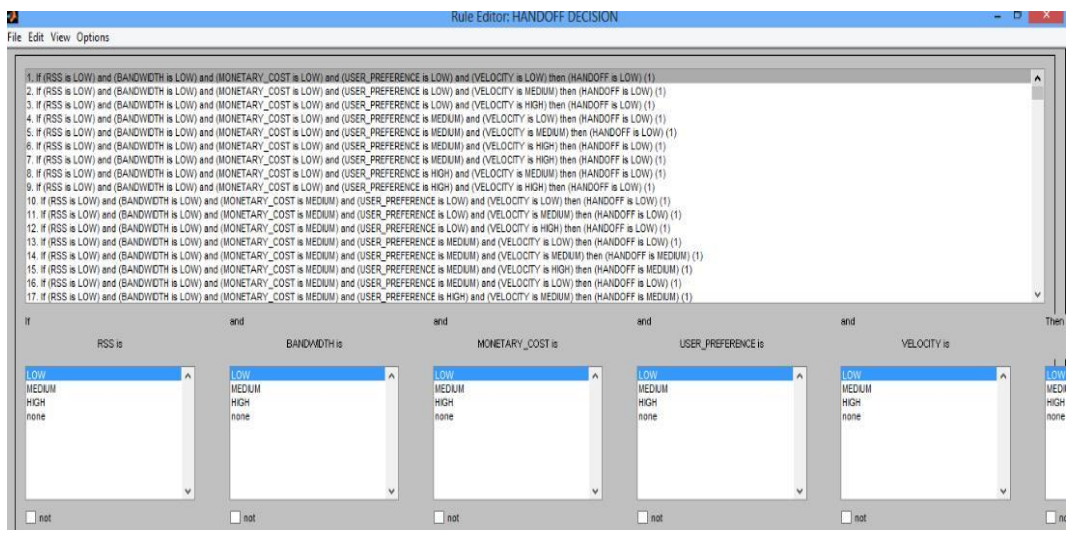

Figure 3: Rule Editor Showing Middle Sets Of Rules

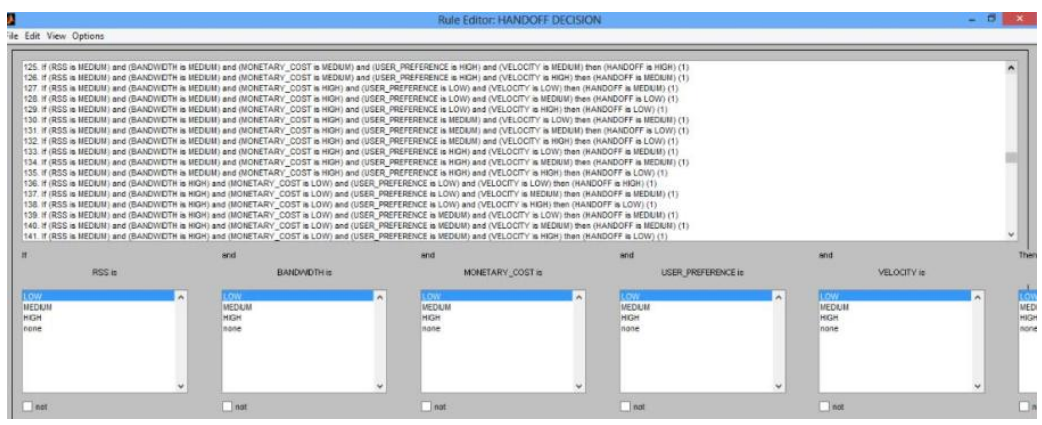

Figure 4: Rule Editor Showing Middle Sets Of Rules 


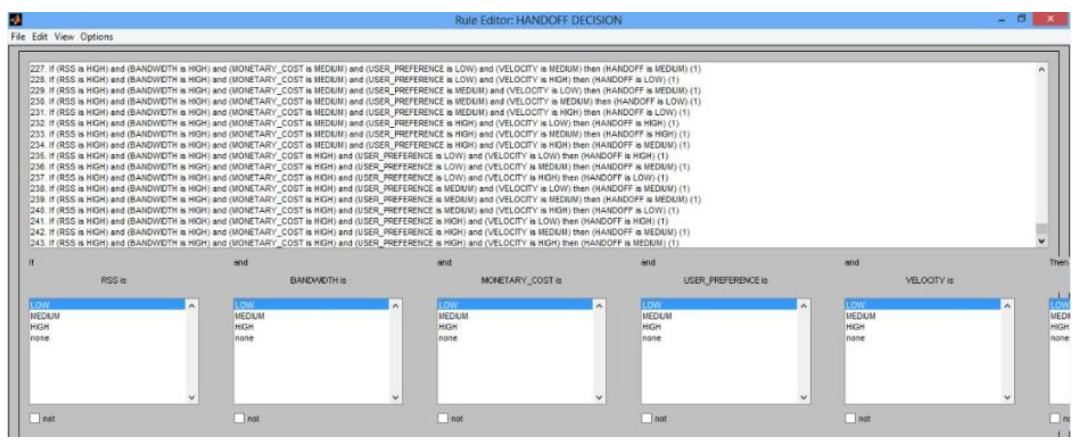

Figure 5: Rule Editor Showing Last Set Of Rules

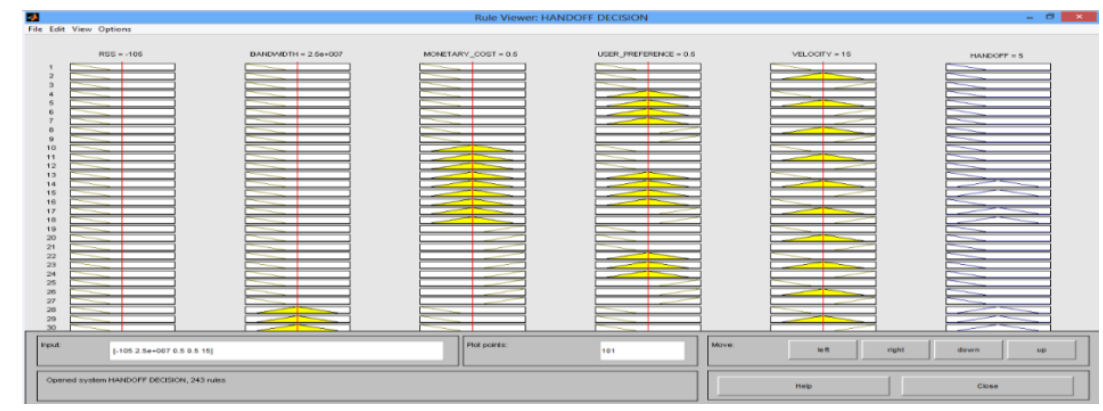

Figure 6: Rule Viewer

\section{DISCUSSION}

As an initial step the decision making is done using fuzzy logic. The first set of the membership function is viewed in the ruler view shown in the Figure 6 from which the variation of input parameters and the output handover decision can be analyzed. The view can be extended till 243 rules and by adjusting the rule from minimum to maximum values the variation of the handover requirement can be viewed and analyzed for the preferred universe of discourse. As rules increases the decision making for handover is more converged for its better performance which reduces the number of handover required and also the delay involved in handover.

\section{CONCLUSION}

The Future 4G wireless mobile communications will completely be heterogeneous networks integrating different wireless access technologies in a complementary manner. Currently proposed algorithms, architectures are restricted to the typical scenarios considering certain networks with conditions. Yet the vertical handover decision has to address innumerable problems in an overlay scenario with multi- criterion, multiple decisions and has to be optimized for better performance which can be done using soft computing techniques.

\section{REFERENCES}

1. C. Chi, X. Cai, R. Hao, F. Liu. Modeling and analys is of handover algorithms , in Proc. IEEE Global

Telecommun. Conf. (GLOBECOM'07), Was hington, DC, USA, November 2007, pp. 4473-4477.

J. McNair \& F. Zhu, June(2004) "Vertical Handovers in Fourth -generation 
Multi-network Environments

", IEEE Wireless Commun. Vol. 11, No. 3, pp 8-15.

Y Lin g, B Yi, Q Zhu. An Improved Vertical Handover Decision Algorithm For Heterogeneous Wireless Network. Wirel. Commun., Networking And Mo Bile Computing, WiCOM 08 ,2008: 1-3

K. Pahlavan, P. Kris hnamurthy, A. Hatami, M. Ylianttila, J. Makela, R. Pichna, and J. Vallstron"

Handover in Hybrid Mobile Data Networks ," IEEE Personal Commun. Magazine, vol. 7, no. 2, 2000,

pp. 34-47.

5. I. Akyildiz, J. Xie, S. Mohanty, A s urvey of mobility management in next-generation all-IP- based wireless systems, IEEE Wirel. Commun. 11 (4) (2004) 16-28.

M. Bernas chi, F. Cacace, G. Iannello, S. Za, and A. Pes cape, "Seamles s internetworking of WLANs

and cellular networks: architecture and performance issues in a mobile IPv6 s cenario," IEEE Wirel.

Commun. Mag., vol. 12, no. 3, pp. 73-80, Jun 2005

7. Ali cephal and celal cekan An Adaptive New Fuzzy Based Vertical Handover Decision Algorithm For Wireless Heterogeneous Networks ,978-14244-8010-6,2000 IEEE 21 ${ }^{\text {st }}$ Annual IEEE s ymposium on Pers onal and Mobile Commun.

8. Abolfazl, Wireless Network Access Selection Algorithm Scheme For Heterogeneous Multimedia Traffic, IETE Networks 2013, Vol 2, is s ue4,PP214-223

9. Qing $\mathrm{He}$,A Novel Vertical handover Decision Algorithm in Heterogeneous Networks , 978- 1-4244-

5849-3/10, IEEE 2010

10. Qingyang Song, Abbas Jamalipour, Network Selection in an Integrated Wireless LAN And UMTS Environment Using Mathematical Modeling And Computing Techniques, IEEE Wireless Communication, June 2005

11. Song, W., Jiang, H., Zhuang, W. and Shen, X. (2005) 'Res ource Management for QoS Support

In Cellular/WLAN Interworking', IEEE Network Magazine, Vol. 19, No. 5, pp. $12-28$.

12. E.Stevens -Navarro,Vincent W.S.Wo ng \& Yuxia Lin,(2007) "A Vertical Handover Decision Algorithm for Heterogeneous Wireless Networks ",In Proc. of Wirel. Commun. and Networking Conf., IEEE ;

doi:10.1109/WCNC. 2007.590.

13. SuKyoung Lee , Kotikalapudi Sriram, Kyungsoo Kim, Yoon Hyuk Kim \& Nada Golmie, "Vertical Handover Decis ion Algorithms for Providing Optimized Performance in Heterogeneous Wireless Networks",IEEE Trans actions on Vehicular Technology. January (2009)

14. M. Stemm and R. Katz, "Vertical handovers in wireles s overlay networks,"Mobile Networks

and Applications, vol. 3, no. 4, pp. 335-350, January 1998.

15. Sunisa Kunarak and Raungrong Suleesathira. "Predictive RSS with Fuzzy Logic Based Vertical

Handover Algorithm in Heterogeneous Wireless Networks ", Proc. of International Symp. on

Commun. and Information Technol. 2010. p. 1235-1240.

16. Tansir Ahmed ,A Context Aware Vertical Handover Decision Algorithm for Multimode Mobile Terminals and its Performance.

17. Vijay.K.Varma, Sudha Rames h, K.Daniel Wong,Jeffery A.Friedhoffer, Mobility Management in

Integrated UMTS/WLAN Networks, 2003 IEEE Transactions .

18. K. Yang, I. Gondal, B. Qiu, L.S. Dooley, Combined SINR Based Vertical 
Mobility Management in 4G Wireless Networks

Handover Algorithm For Next Generation Heterogeneous Wireless Networks .Procs of the 2007 IEEE Global Telecommun. Co nf. (GLOBECOM '07), Washington, DC, USA, November 2007.

19. Y.H.Wang,CHs u, KF.Huang,W C Huang. "Handover Decision Scheme with Guaranteed QoS in

Heterogeneous Network .First IEEE International Conf. on Ubi-Media Computing, 2008:138143.

20. A.H. Zahran, B. Liang, Performance Evaluation Framework for Vertical Handover Algorithms in Heterogeneous Networks, Proc. of the 2005 IEEE International Conf. on Commun. (ICC'05), Seoul, Korea, May 2005, pp. 173-178.

Received: February 03, 2016; Accepted: July 14, 2016 